\title{
Parameter dependence and stability of guided TE-waves in a lossless nonlinear dielectric slab structure
}

\author{
Hans Werner Schürmann ${ }^{1, *}$ and Valery Serov ${ }^{2, \dagger}$ \\ ${ }^{1}$ Department of Physics \\ University of Osnabrück, Germany \\ ${ }^{2}$ Department of Mathematical Sciences \\ University of Oulu, Finland
}

\begin{abstract}
The nonlinear Schrödinger equation is the basis of the traditional stability analysis of nonstationary guided waves in a nonlinear three-layer slab structure. The stationary (independent of the propagation distance) solutions of the nonlinear Schrödinger equation are used as "initial data" in this analysis. In the present paper, we propose a method to investigate the dependence of these solutions on the experimental parameters and discuss their stability with respect to the parameters. The method is based on the phase diagram condition (PDC) and compact representation (in terms of Weierstrass' elliptic function and its derivative) of the dispersion relation (DR). The problem's parameters are constrained to certain regions in parameter space by the PDC. Dispersion curves inside (or at boundaries) of these regions correspond to possible physical solutions of Maxwell's equations as "start" solutions for a traditional stability analysis. Numerical evaluations of the PDC, DR, and power flow including their parameter dependence are presented.
\end{abstract}




\section{INTRODUCTION}

Investigating stability of electromagnetic fields in waveguides (linear or nonlinear) represents an important issue in physics and applied mathematics. In nonlinear waveguide theory, stability of the transverse electric field $\mathcal{E}_{y}(x, z, t)$ (in a planar three-layer structure (see Fig.1)) usually is studied by assuming an ansatz (to solve Maxwell's equations)

$$
\mathcal{E}_{y}(x, z, t)=\tilde{E}_{y}(x, z, \gamma) e^{i(\gamma z-\omega t)}
$$

leading to Helmholtz equations for $\tilde{E}_{y}(x, z, \gamma)$ valid in the three layers. If the nonlinear part of the permittivity is small compared with the linear one the well-known [1] "Slowly Varying Envelope Approximation" $\left(\left|\partial_{z} \tilde{E}_{y}\right| \ll\left|\gamma \tilde{E}_{y}\right|\right)$ can be applied. It approximates the Helmholtz equations by nonlinear Schrödinger equations (NLSEs)

$$
2 i \gamma \frac{\partial \tilde{E}_{y}}{\partial z}+\frac{\partial^{2} \tilde{E}_{y}}{\partial x^{2}}-\left(\gamma^{2}-\epsilon\left(\tilde{E}_{y}^{2}\right)\right) \tilde{E}_{y}=0,
$$

which are used as the basis for a stability analysis with respect to the propagation distance $z$. From the physical point of view the stability problem is how an initial field profile (at $z=0$, say) $\tilde{E}_{y}(x, 0, \gamma)$ evolves with increasing $z$. The propagation constant $\gamma$, as solution of the dispersion relation (DR), depends on the thickness $h$ of the film and on the (material) parameters of the problem. A stability analysis that yields (e.g.) a stable ( $z$-independent) profile $E_{y}\left(x, \gamma_{0}\right)$ with a certain propagation constant $\gamma_{0}$, may lead, for slightly different parameters, to a propagation constant $\gamma$ not in the vicinity of $\gamma_{0}$. In general, this implies that the profile $E_{y}\left(x, \gamma_{0}\right)$ and $E_{y}(x, \gamma)$ differ considerably, indicating instability. Thus, obviously, it is important, for physical application, to know the parameter dependence of the propagation constant $\gamma$. From the mathematical point of view the stability problem is studied by introducing a perturbation function $f(x, z, \gamma)$ and setting $\tilde{E}_{y}(x, z, \gamma)=E_{y}(x, \gamma)+f(x, z, \gamma)$. The NLSE (2) can be linearised leading to a system of two coupled differential equations [2] that are rewritten as an eigenvalue problem for $f(x, z, \gamma)$ (with two operators). The growth rate of $f(x, z, \gamma)$ is studied by analysing the spectrum of the operators [2], [4]. For discrete positive imaginary eigenvalues, $f(x, z, \gamma)$ decays to zero as $z \rightarrow \infty[2]$, so that, in this sense, $E_{y}(x, \gamma)$ is stable. It is important to note that in this approach, applied in numerous articles [2] - [17], the propagation constant $\gamma$ is obtained by assuming $\tilde{E}_{y}(x, z, \gamma)$ constant with respect to $z$, i.e., $\tilde{E}_{y}(x, z, \gamma)=E_{y}(x, \gamma)[2]$. Hence, the eigenvalues and thus the growth rate of $f(x, z, \gamma)$ depend on $E_{y}(x, \gamma)$. As is well known (see, e.g., [18], [19]), $E_{y}(x, \gamma)$ can be singular or a (real) propagation constant $\gamma$, may not exist for certain parameters $p_{i} \in\left\{h, J_{0}, \bar{\epsilon}_{\nu}\right\}$. Furthermore, needless to say, that the decay properties of the perturbation eigenfunction $f$ depends on the parameters $p_{j}$.

Based on the phase diagram analysis [20] conditions for solvability and existence of solutions $\gamma\left(p_{i} ; p_{j}\right)$ [21] of the DR have been derived [22] (b). With respect to the traditional stability analysis, sketched before, they are appropriate to describe nonnegative and bounded ("physical") intensities $J(x, \gamma)=E_{y}^{2}(x, \gamma)$. In connection with stability, there are three possibilities for $\gamma=\gamma\left(p_{i} ; p_{j}\right)$ to depend on a variation $\delta p_{j}$. First, the resulting mode $\tilde{\gamma}\left(p_{i} ; p_{j}+\delta p_{j}\right)$ can be inconsistent with the PDC. Second, $\tilde{\gamma}\left(p_{i} ; p_{j}+\delta p_{j}\right)$ may satisfy the PDC with $\tilde{\gamma} \not \gamma$ as $\delta p_{j} \rightarrow 0$. Third, again with PDC satisfied, $\tilde{\gamma} \rightarrow \gamma$ as $\delta p_{j} \rightarrow 0$.

The paper is organized as follows. Section II presents the problem. Section III is devoted to the solutions of the basic nonlinear differential equations, and to the dispersion relations 


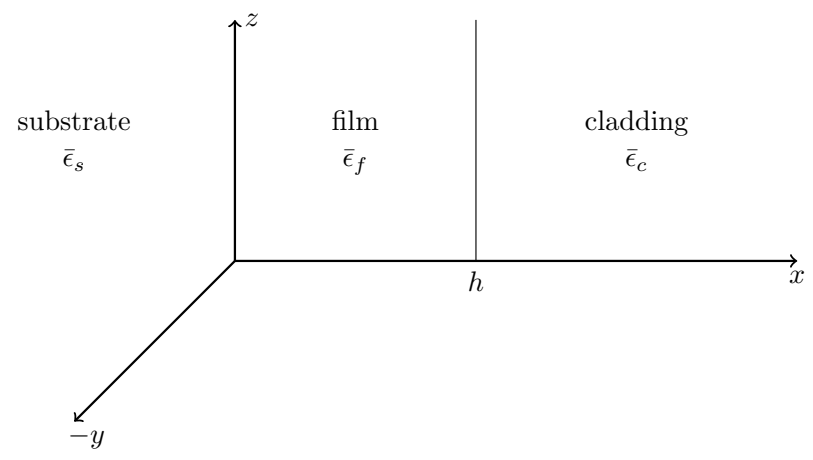

FIG. 1. Geometry of the problem

with its solvability conditions. The results of Section III are applied for certain parameters $\epsilon_{\nu}, a_{\nu}, h$ in Section IV. The paper concludes with a summary and some remarks in Section $\mathrm{V}$.

\section{STATEMENT OF THE PROBLEM}

We consider a planar waveguide structure with lossless, isotropic, nonmagnetic homogeneous material and a permittivity $\epsilon$ according to

$$
\epsilon=\left\{\begin{array}{r}
\bar{\epsilon}_{s}=\epsilon_{s}+a_{s}\left|\mathcal{E}_{y}\right|^{2}, \quad x<0 \\
\bar{\epsilon}_{f}=\epsilon_{f}+a_{f}\left|\mathcal{E}_{y}\right|^{2}, \quad 0 \leq x \leq h \\
\bar{\epsilon}_{c}=\epsilon_{c}+a_{c}\left|\mathcal{E}_{y}\right|^{2}, \quad x>h
\end{array}\right.
$$

with $\epsilon_{\nu}, a_{\nu}, \nu=s, f, c$, (see Fig.1) real and constant.

Assuming $\tilde{E}_{y}(x, z, \gamma)$ and propagation constant $\gamma$ to be real and inserting ansatz (1) with $\tilde{E}_{y}(x, z, \gamma)=E_{y}(x, \gamma)$ into Maxwell's equations we obtain

$$
\frac{d^{2} E_{y}(x, \gamma)}{d x^{2}}=\left\{\begin{array}{rr}
\left(\gamma^{2}-\omega^{2} \bar{\epsilon}_{s} \mu_{0}\right) E_{y}(x, \gamma), \quad x<0 \\
\left(\gamma^{2}-\omega^{2} \bar{\epsilon}_{f} \mu_{0}\right) E_{y}(x, \gamma), \quad 0 \leq x \leq h \\
\left(\gamma^{2}-\omega^{2} \bar{\epsilon}_{c} \mu_{0}\right) E_{y}(x, \gamma), \quad x>h
\end{array}\right.
$$

With $k_{0}^{2}=\omega^{2} \mu_{0} \epsilon_{0}$ and scaling $\gamma$ and $x$ by $k_{0}, \epsilon$ by $\epsilon_{0}$, Helmholtz equations (4) can be written as

$$
\frac{d^{2} E(x, \gamma)}{d x^{2}}=\left\{\begin{array}{r}
\left(\gamma^{2}-\bar{\epsilon}_{s}\right) E(x, \gamma), \quad x<0 \\
\left(\gamma^{2}-\bar{\epsilon}_{f}\right) E(x, \gamma), \quad 0 \leq x \leq h \\
\left(\gamma^{2}-\bar{\epsilon}_{c}\right) E(x, \gamma), \quad x>h
\end{array}\right.
$$

where $E(x, \gamma)$ denotes $E_{y}(x, \gamma)$ with scaled arguments. Inserting $\bar{\epsilon}_{\nu}$ according to Eqs.(3) into Eqs.(5), multiplying by $E^{\prime}$, and integrating (with respect to $E$ ) we get

$$
\left(\frac{d J(x, \gamma)}{d x}\right)^{2}=\left\{\begin{array}{r}
-2 a_{s} J^{3}(x, \gamma)+4\left(\gamma^{2}-\epsilon_{s}\right) J^{2}(x, \gamma)+4 C_{s} J(x, \gamma):=R_{s}(J), \quad x<0 \\
-2 a_{f} J^{3}(x, \gamma)+4\left(\gamma^{2}-\epsilon_{f}\right) J^{2}(x, \gamma)+4 C_{f} J(x, \gamma):=R_{f}(J), \quad 0 \leq x \leq h, \\
-2 a_{c} J^{3}(x, \gamma)+4\left(\gamma^{2}-\epsilon_{c}\right) J^{2}(x, \gamma)+4 C_{c} J(x, \gamma):=R_{c}(J), \quad x>h,
\end{array}\right.
$$


where $J(x, \gamma)=E^{2}(x, \gamma)$, and $C_{\nu}$ are the integration constants. The problem is, first, to find propagation constant $\gamma$ associated to physical solutions $J_{\nu}(x, \gamma)$ of Eqs.(6) that satisfy radiation conditions at infinity

$$
E(x, \gamma) \rightarrow 0, \quad \frac{d E(x, \gamma)}{d x} \rightarrow 0, \quad|x| \rightarrow \infty
$$

second, to analyse the parameter dependence of $\gamma$, and thus the stability of solutions $J_{\nu}(x, \gamma)$.

\section{SOLUTION}

Apart from $J(x, \gamma)=$ constant the solutions of Eqs.(6) are [23], [22]:

$$
\begin{gathered}
J_{s \pm}(x, \gamma)=\frac{J_{0}}{\left(\cosh \left(x \sqrt{\gamma^{2}-\epsilon_{s}}\right) \mp \sqrt{1-\frac{a_{s} J_{0}}{2\left(\gamma^{2}-\epsilon_{s}\right)}} \sinh \left(x \sqrt{\gamma^{2}-\epsilon_{s}}\right)\right)^{2}}, \quad x<0, \\
J_{f \pm}(x, \gamma)=J_{0}+ \\
+\frac{\frac{1}{2} R_{f}^{\prime}\left(J_{0}\right)\left(\wp-\frac{1}{24} R_{f}^{\prime \prime}\left(J_{0}\right) \pm \wp^{\prime} \sqrt{R_{f}\left(J_{0}\right)}+\frac{1}{24} R_{f}\left(J_{0}\right) R_{f}^{\prime \prime \prime}\left(J_{0}\right)\right.}{2\left(\wp-\frac{1}{24} R_{f}^{\prime \prime}\left(J_{0}\right)\right)^{2}-\frac{1}{48} R_{f}\left(J_{0}\right) R_{f}^{\prime \prime \prime \prime}\left(J_{0}\right)}, \quad 0 \leq x \leq h,
\end{gathered}
$$

or, equivalently,

$$
\begin{gathered}
J_{f \pm}(x, \gamma)=J_{0}-\frac{9 a_{f} J_{0}^{2}-12\left(\gamma^{2}-\epsilon_{f}\right) J_{0}-6 C_{f}}{6 \wp\left(x ; g_{2}, g_{3}\right)+3 a_{f} J_{0}-2\left(\gamma^{2}-\epsilon_{f}\right)}- \\
-\frac{18\left(a_{f} J_{0}^{3}-2\left(\gamma^{2}-\epsilon_{f}\right) J_{0}^{2}-2 C_{f} J_{0}\right)}{\left(6 \wp\left(x ; g_{2}, g_{3}\right)+3 a_{f} J_{0}-2\left(\gamma^{2}-\epsilon_{f}\right)\right)^{2}} \pm \frac{18 \wp^{\prime}\left(x ; g_{2}, g_{3}\right) \sqrt{-2 a_{f} J_{0}^{3}+4\left(\gamma^{2}-\epsilon_{f}\right) J_{0}^{2}+4 C_{f} J_{0}}}{\left(6 \wp\left(x ; g_{2}, g_{3}\right)+3 a_{f} J_{0}-2\left(\gamma^{2}-\epsilon_{f}\right)\right)^{2}}
\end{gathered}
$$

with $g_{2}$ and $g_{3}$ according to Eqs.(12), and with derivative

$$
\begin{gathered}
\partial_{x} J_{f \pm}(x, \gamma)=\frac{\frac{1}{2} R_{f}^{\prime}\left(J_{0}\right) \wp^{\prime} \pm\left(6 \wp^{2}-\frac{g_{2}}{2}\right) \sqrt{R_{f}\left(J_{0}\right)}}{2\left(\wp-\frac{1}{24} R_{f}^{\prime \prime}\left(J_{0}\right)\right)^{2}-\frac{1}{48} R_{f}\left(J_{0}\right) R_{f}^{\prime \prime \prime \prime}\left(J_{0}\right)} \\
-\frac{2 R_{f}^{\prime}\left(J_{0}\right) \wp^{\prime}\left(\wp-\frac{1}{24} R_{f}^{\prime \prime}\left(J_{0}\right)\right)^{2} \pm 4\left(\wp-\frac{1}{24} R_{f}^{\prime \prime}\left(J_{0}\right)\right)\left(\wp^{\prime}\right)^{2} \sqrt{R_{f}\left(J_{0}\right)}}{\left(2\left(\wp-\frac{1}{24} R_{f}^{\prime \prime}\left(J_{0}\right)\right)^{2}-\frac{1}{48} R_{f}\left(J_{0}\right) R_{f}^{\prime \prime \prime \prime}\left(J_{0}\right)\right)^{2}} \\
-\frac{\frac{1}{6} \wp^{\prime}\left(\wp-\frac{1}{24} R_{f}^{\prime \prime}\left(J_{0}\right)\right) R_{f}\left(J_{0}\right) R_{f}^{\prime \prime \prime}\left(J_{0}\right)}{\left(2\left(\wp-\frac{1}{24} R_{f}^{\prime \prime}\left(J_{0}\right)\right)^{2}-\frac{1}{48} R_{f}\left(J_{0}\right) R_{f}^{\prime \prime \prime \prime}\left(J_{0}\right)\right)^{2}}, \quad 0 \leq x \leq h, \\
J_{c \pm}(x, \gamma)=\frac{J^{(h)}}{\left(\cosh \left((x-h) \sqrt{\gamma^{2}-\epsilon_{c}}\right) \mp \sqrt{1-\frac{a_{c} J^{(h)}}{2\left(\gamma^{2}-\epsilon_{c}\right)}} \sinh \left((x-h) \sqrt{\gamma^{2}-\epsilon_{c}}\right)\right)^{2}}, \quad x>h,
\end{gathered}
$$


where $\wp\left(x ; g_{2}, g_{3}\right)$ denotes Weierstrass' elliptic function, and $J_{0}, J^{(h)}$ have been chosen so that intensities are continuous at $x=0$ and $x=h$, respectively. The invariants $g_{2}, g_{3}$ of $\wp\left(x ; g_{2}, g_{3}\right)$ are given by

$$
\left\{\begin{array}{r}
g_{2}=2 a_{f} C_{f}+\frac{4}{3}\left(\gamma^{2}-\epsilon_{f}\right)^{2}, \\
g_{3}=\frac{2}{3} a_{f} C_{f}\left(\epsilon_{f}-\gamma^{2}\right)-\frac{8}{27}\left(\gamma^{2}-\epsilon_{f}\right)^{3}
\end{array}\right.
$$

In Eqs.(9) and (10) the prime denotes differentiation with respect to $J$ for $R_{f}(J)$ and differentiation with respect to $x$ for $\wp\left(x ; g_{2}, g_{3}\right)$. In deriving Eqs.(8) and (11), $C_{s}=C_{c}=0$ has been used (according to condition (7) applied to $R_{\nu}(J), \nu=s, c$, written in terms of $E$ ) and, as established below, $a_{s} \geq 0, a_{c} \geq 0$. Integration constant $C_{f}$ in Eqs.(6) is determined by the transmission conditions at $x=0$ or at $x=h$.

The field components $E_{y}$ and $H_{z}$ are continuous at the interfaces between the layers. This implies continuity of $J(x, \gamma)$ and $\partial_{x} J(x, \gamma)$. By using Eqs.(8)-(11) we obtain $J_{f \pm}\left(0, \gamma ; p_{j}\right)=$ $J_{s \pm}\left(0, \gamma ; p_{j}\right)=J_{0}$ and

$$
\begin{gathered}
\left.\partial_{x} J_{f \pm}\left(x, \gamma ; p_{j}\right)\right|_{x=0}=\mp \sqrt{-2 a_{f} J_{0}^{3}+4\left(\gamma^{2}-\epsilon_{f}\right) J_{0}^{2}+4 C_{f} J_{0}}, \\
\left.\partial_{x} J_{s \pm}\left(x, \gamma ; p_{j}\right)\right|_{x=0}=\mp \sqrt{-2 a_{s} J_{0}^{3}+4\left(\gamma^{2}-\epsilon_{s}\right) J_{0}^{2}} .
\end{gathered}
$$

By evaluation of the transmission condition $\left.\partial_{x} J_{f \pm}\left(x, \gamma ; p_{j}\right)\right|_{x=0}=\left.\partial_{x} J_{s \pm}\left(x, \gamma ; p_{j}\right)\right|_{x=0}$ according to Eqs.(13) and (14), integration constant $C_{f}$ in Eqs.(6) is determined (independently on sign combination) as

$$
C_{f}=\frac{J_{0}^{2}}{2}\left(a_{f}-a_{s}\right)+J_{0}\left(\epsilon_{f}-\epsilon_{s}\right) .
$$

To find the dispersion relation (DR), continuity of $J(x, \gamma)$ and $\partial_{x} J(x, \gamma)$ at $x=h$ must be evaluated. We obtain from Eqs.(9) and (11)

$$
J_{f \pm}\left(h, \gamma ; p_{j}\right)=J_{c \pm}\left(h, \gamma ; p_{j}\right)=J^{(h)}\left(p_{j}\right),\left.\quad \partial_{x} J_{f \pm}\left(x, \gamma ; p_{j}\right)\right|_{x=h}=\left.\partial_{x} J_{c \pm}\left(h, \gamma ; p_{j}\right)\right|_{x=h} .
$$

Squares in the last equation (16) yields $R_{f}\left(J^{(h)}\left(p_{j}\right)\right)=R_{c}\left(J^{(h)}\left(p_{j}\right)\right)$ leading to

$$
\frac{\left(J^{(h)}\left(p_{j}\right)\right)^{2}}{2}\left(a_{f}-a_{c}\right)+J^{(h)}\left(p_{j}\right)\left(\epsilon_{f}-\epsilon_{c}\right)-C_{f}=0 .
$$

Thus, if the discriminant $D$ of Eq.(17) is nonnegative,

$$
D=\left(\epsilon_{f}-\epsilon_{c}\right)^{2}+2 C_{f}\left(a_{f}-a_{c}\right) \geq 0,
$$

two real roots $(D>0)$ or one double real root $(D=0)$ exist. We need the positive roots (at least one positive root) which must be consistent with Eqs.(16). The derivative $\left.\partial_{x} J_{f \pm}\left(x, \gamma ; p_{j}\right)\right|_{x=h}$ in Eqs.(16) can be evaluated straightforwardly (see Eq.(10)). Due to Eq.(16), derivative $\left.\partial_{x} J_{c \pm}\left(x, \gamma ; p_{j}\right)\right|_{x=h}$ is given by

$$
\left.\partial_{x} J_{c \pm}\left(x, \gamma ; p_{j}\right)\right|_{x=h}=\mp \sqrt{-2 a_{c}\left(J_{f \pm}\left(h, \gamma ; p_{j}\right)\right)^{3}+4\left(\gamma^{2}-\epsilon_{c}\right)\left(J_{f \pm}\left(h, \gamma ; p_{j}\right)\right)^{2}} .
$$

Thus Eq.(16) leads to the dispersion relation

$$
\left.\partial_{x} J_{f \pm}\left(x, \gamma ; p_{j}\right)\right|_{x=h}= \pm \sqrt{-2 a_{c}\left(J_{f \pm}\left(h, \gamma ; p_{j}\right)\right)^{3}+4\left(\gamma^{2}-\epsilon_{c}\right)\left(J_{f \pm}\left(h, \gamma ; p_{j}\right)\right)^{2}}
$$


The sign of the LHS depends on the choice of $J_{f+}$ or $J_{f-}$ and on $\gamma$ and $h$. Thus, for evaluation in general, both signs of the RHS of Eq.(20) must be taken into account (in Section IV, to simplify, we have assumed $a_{s}=a_{c}=0$, so that the RHS is negative). Equation (20) represents a compact relation between the propagation constant $\gamma$ and the parameters of the problem. If, e.g., the intensity at $x=0, J_{f}(0)=J_{s}(0)=J_{0}$ and the material parameters are fixed, Eq.(20) relates $\gamma$ and $h$. For evaluation of the DR only nonnegative and bounded intensities $J_{f \pm}\left(x, \gamma ; p_{j}\right)$ are appropriate. To find them, it is convenient to consider phase diagrams (PDs) $\left\{R_{\nu}(J), J\right\}$. A little thought shows that ten PDs correspond to physical $J_{f}$ and one PD to physical $J_{s, c}$, as depicted in Fig.2. As is well known (see [22(a)]), the hatched intervals, labelled $I_{1}$, are related to bounded solutions $J_{f}$ (oscillatory for PDs (a), (b), (f), solitary for PDs (g), (i)). The dashed-line intervals $I_{2}$ correspond to unbounded solutions $J_{f}$ (see $[22(\mathrm{a})]$ ) (due to Eq.(9) $J_{f}$ is meromorphic in $x$ with poles $x_{i}$ ). If $\min \left(x_{i}\right)=\bar{x}>0$ and $\bar{x}>h$, then $J_{f}$ is bounded for $0 \leq x \leq h$ and can be used for evaluation of the DR also (as the solutions $J_{f}$ for PDs (a), (b), (f), (g), (i)). For simplicity however, we consider in the following only bounded solutions $J_{f} \in I_{1}$ associated to PDs (a), (b), (f), (g), (i) (this restriction seems irrelevant for elucidating the approach). Considering solutions $J_{f} \in I_{1}$, the corresponding PDs are suitable, first, to enable a test for existence of solutions $\gamma\left(p_{i}, p_{j}\right)$ of the DR, and second to describe the parameter dependence of $\gamma\left(p_{i}, p_{j}\right)$. Evidently, $J_{0}$ and $J^{(h)}$, with $J^{(h)}$ according Eqs.(16)-(18) must be located in intervals $I_{1}$ in one of the PDs $\left\{R_{f}(J), J\right\}$ and in intervals $I_{1}$ of $\mathrm{PDs}\left\{R_{s, c}(J), J\right\}$. This requirements can be written as

$$
\begin{gathered}
J=0, \quad J_{1,2}=\frac{\gamma^{2}-\epsilon_{f} \mp \sqrt{\left(\gamma^{2}-\epsilon_{f}\right)^{2}+2 a_{f} C_{f}}}{a_{f}}, \\
\left\{\begin{array}{r}
J_{0} \in\left[j_{1}, j_{2}\right] \cap\left(0, \frac{2\left(\gamma^{2}-\epsilon_{s}\right)}{a_{s}}\right] \neq \emptyset, \quad J^{(h)} \in\left[j_{1}, j_{2}\right] \cap\left[0, \frac{2\left(\gamma^{2}-\epsilon_{c}\right)}{a_{c}}\right] \neq \emptyset, \\
\text { with } \quad a_{s} \geq 0, a_{c} \geq 0 \quad \text { and } \quad \gamma^{2}-\epsilon_{s}>0, \gamma^{2}-\epsilon_{c}>0,
\end{array}\right.
\end{gathered}
$$

where $j_{1}, j_{2} \in\left\{J=0, J_{1,2}\right\}$ with $0, J_{1}, J_{2}$ as the roots of $R_{f}(J)=0$. Thus, $j_{1}, j_{2}$ are the lower and upper bounds of intervals $I_{1}$ of $\mathrm{PDs}\left\{R_{f}(J), J\right\}$, respectively. Solutions $J_{f}$ must be associated to (at least) one of the PDs. Thus, evaluation of conditions (22), with $J_{1,2}$ and $J_{1,2}^{(h)}$ according to Eq.(17), leads (applying some algebra) to a lengthy compact condition, denoted the phase diagram condition (PDC), presented in Appendix A (in deriving the PDC, for simplification, we have assumed $a_{s}=a_{c}=0$, as mentioned above). In parameter space, the PDC defines regions (referred to as PDC regions in the following) that represent parameter sets corresponding to bounded nonnegative solutions $J_{\nu \pm}\left(x, \gamma ; p_{j}\right)$.

Returning to the DR (20), we first note that $J_{f \pm}\left(h, \gamma ; p_{j}\right)$ is continuously differentiable w.r.t. $h, \gamma, p_{j}$ if the PDC is satisfied (due to the PDC, denominators in Eqs.(9) and (10) do not vanish, and, since $\wp\left(h, g_{2}, g_{3}\right)$ is holomorphic in $g_{2}\left(\gamma, p_{j}\right), g_{3}\left(\gamma, p_{j}\right)$ (see [24], 18.5.1-3), $J_{f \pm}\left(x, \gamma ; p_{j}\right)$ is continuously differentiable). By varying $\{h, \gamma\}, p_{j}$ fixed, and $\gamma, p_{j}$ satisfying the PDC, both sides of the DR (20) are varying continuously, and a contourplot w.r.t. to $\{h, \gamma\}$ can serve as a test for existence of physical solutions $\{h, \gamma\}$. Due to analytic properties mentioned, this procedure also works if $h=h_{0}$ is fixed, and a certain parameter $p_{i}$ is variable. This leads to a representation $\left\{p_{i}, \gamma\right\}$ which describes the dependence of $\gamma$ on $p_{i}$. We note, secondly, that in this context the PDC (again) plays the central role since it restricts the domain of $p_{i}$ (if $p_{i}=h$, there is no restriction). Due to $a_{c}=0$, additionally to the PDC, $\left.\partial_{x} J_{f-}\left(x, \gamma ; p_{j}\right)\right|_{x=h}<0$ must hold. As a consequence, dispersion curves $\gamma\left(p_{i}\right)$ must be located in regions defined by PDC and $\left.\partial_{x} J_{f-}\left(x, \gamma ; p_{j}\right)\right|_{x=h}<0$. - Examples are presented in the following Section. 


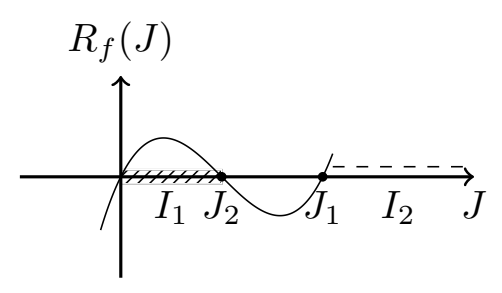

(a)

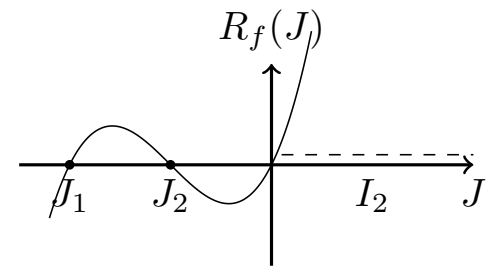

(b)

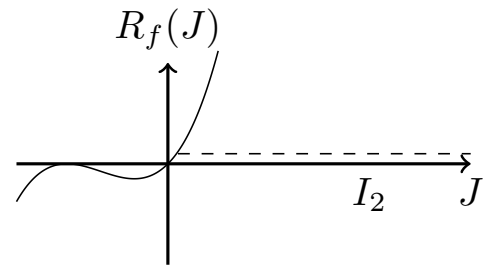

(c)

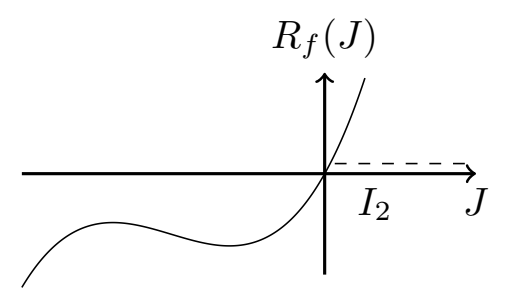

(d)

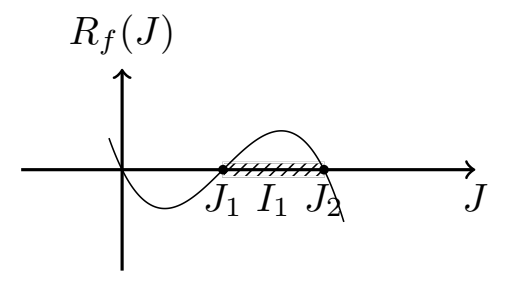

(e)

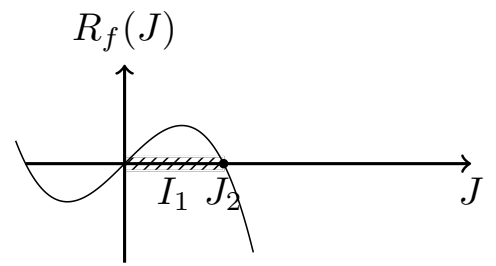

(f)

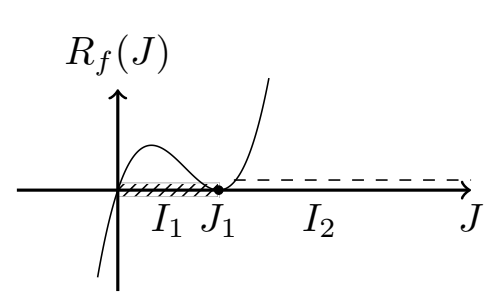

(g)

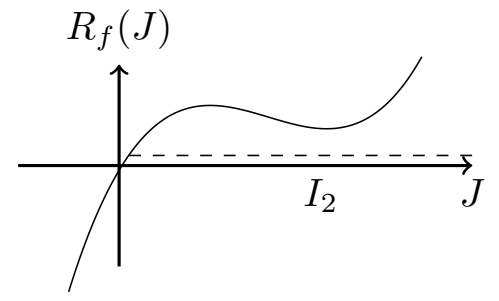

(h)

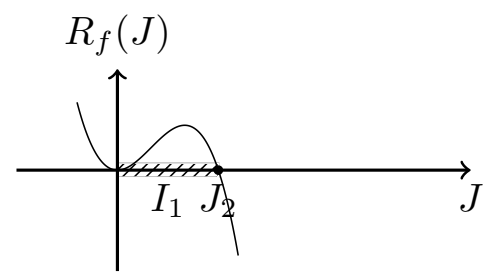

(i)

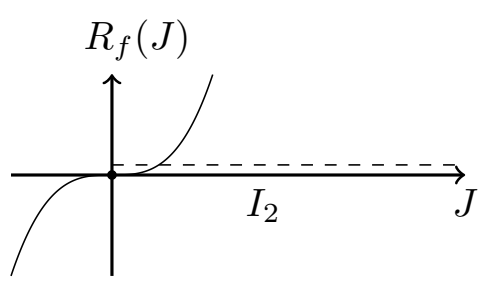

(j)

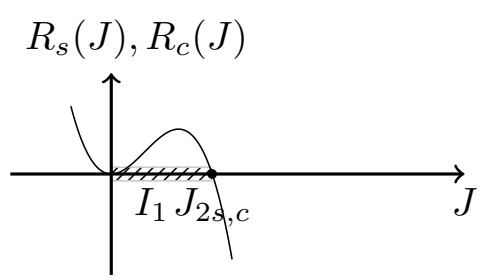

(k)

FIG. 2. Phase diagrams for solutions $J_{\nu \pm}\left(x, \gamma ; p_{j}\right), \nu=s, f, c: J_{\nu \pm}\left(x, \gamma ; p_{j}\right) \in I_{1}$ bounded solutions, $J_{f \pm}\left(x, \gamma ; p_{j}\right) \in I_{2}$ bounded or unbounded depending on $h$ (comments in the text)

The foregoing approach to investigate the parameter dependence and stability of physical solutions $J(x, \gamma)$ of Eqs.(6) can be summarized as follows.

(I) If parameters $\epsilon_{\nu}, a_{\nu}$ are prescribed, condition (18) restricts $J_{0}>0$ w.r.t. $\epsilon_{\nu}, a_{\nu}\left(J_{0}\right.$ cannot be chosen arbitrary if the material parameters are prescribed). Subject to this restriction, only one positive root $J^{(h)}$ exists (if $\frac{2 C_{f}}{a_{c}-a_{f}}<0$ ) or two positive roots $J_{1,2}^{(h)}$ (if $\left.\frac{2 C_{f}}{a_{c}-a_{f}}>0\right)$. Both cases must be considered for evaluation of the dispersion relation.

(II) Using $J_{0}, J^{(h)}$ according to (I), the PDC represents a necessary restriction for $\gamma$ that must be taken into account for evaluation of the dispersion relation. Finally, $\gamma, J_{0}, J^{(h)}$ must be such that one of PDs of Fig.2 (a), (b), (f), (g), (i) occurs.

(III) Solutions $\gamma=\gamma\left(p_{i}\right)$ (due to (I) and (II)) are associated to intensities $J_{f \pm}\left(x, \gamma ; p_{j}\right.$ ) that are continuously differentiable w.r.t. $x, \gamma, p_{j}$, so that the transmission conditions can be evaluated, leading to $C_{f}$ according to Eq.(15) (continuity of $J_{f \pm}\left(x, \gamma ; p_{j}\right)$ and $\partial_{x} J_{f \pm}\left(x, \gamma ; p_{j}\right)$ 
at $x=0$ ) and to the dispersion relation (20) (continuity of $\partial_{x} J_{f \pm}\left(x, \gamma ; p_{j}\right)$ at $x=h$ ).

(IV) The PDC defines subspaces in parameter space (PDC regions). Solutions $\gamma\left(p_{i} ; p_{j}\right)$ (if they exist) are associated to physical solutions $J(x, \gamma)$ of Eqs.(6), if the PDC is satisfied.

Referring to the possibilities mentioned in Section I, a variation $p_{j}+\delta p_{j}$ of a physical solution $\gamma\left(p_{i} ; p_{j}\right)$ may lead to $\tilde{\gamma}\left(p_{i} ; p_{j}+\delta p_{j}\right)$ that is not consistent with the PDC. It seems suitable to denote such $\tilde{\gamma}\left(p_{i} ; p_{j}+\delta p_{j}\right)$ as "basically unstable" with respect to the parameter $p_{j}$. The other possibilities refer to dispersion curves within the PDC region, where bifurcation points $\left(\partial_{h}\left(p_{i}\right)=0, \partial_{h}^{2}\left(p_{i}\right) \neq 0\right)$ occur. At a bifurcation point $\gamma_{B}$ the behaviour of the propagation constant $\gamma$ is described by $\delta \gamma / 0$ as $\delta p_{j} \rightarrow 0$ (it depends on the sign of $\delta p_{j}$ whether two values of $\gamma$ exist in the vicinity of $\gamma_{B}$ or one $\gamma$ on a different branch of the dispersion curve). For the third possibility $\left(\delta \gamma \rightarrow 0\right.$ as $\left.\delta p_{j} \rightarrow 0\right)$ it can be shown, due to the continuity of $\gamma=\gamma\left(p_{i}\right)$, that the intensity patterns are continuous. Usually, $\gamma\left(p_{i} ; p_{j}\right)$ is denoted as "stable" in this case while it is called "bistable" or "unstable" at bifurcation points (see examples in Section IV).

\section{APPLICATIONS}

Before we elucidate the (mathematical) results of the preceding Section by numerical simulations it seems suitable to point out their physical significance: The above results concentrate on the "stationary" electromagnetic waves $\tilde{E}_{y}(x, \gamma) e^{i(\gamma z-\omega t)}$ according to Eq.(1) and their consistency with the PDC. The waves are characterized by a transverse profile that is independent of both time and distance $z$, and by a definite frequency $\omega$ of oscillation. From a physical point of view, a fundamental concern about these waves is whether they can be existed in a realistic situation. This concern is related to the question how critical are the values of the experimental parameters with respect to the existence of a particular stationary wave. The claim of the present paper is that the PDC together with the DR (20) are suitable to give a partial answer to this question. If a physical solution of the DR, consistent with the PDC, exists, and is stable or bistable with respect to the experimental parameters, a traditional stability analysis with respect to the propagation distance $z$ (either using the method of eigenvalues or numerical simulation) makes sense. It is useful to check PDC and DR, if certain parameters are prescribed, before starting a traditional stability analysis. Numerical results of traditional stability investigations, as shown in the literature (see, e.g., [2, Fig.3], [3, Fig.16]) indicate that it depends on the particular parameters of the stationary solution whether it remains stable over a certain range $z$ or not. Thus, it must be noted with regards to the following numerical simulations that the DR together with the PDC are only necessary for a traditional stability analysis.

As a first example, we consider a slab structure with parameters

$$
\left\{p_{j}\right\}=\left\{a_{s}=a_{c}=0, a_{f}=-0.01, \epsilon_{f}=9, \epsilon_{s}=1, \epsilon_{c}=4, J_{0}=1\right\}
$$

Due to $a_{s}=0$, and using Eqs.(8)-(9) it turns out that only the transmission condition $\left.\partial_{x} J_{f-}\left(x, \gamma ; p_{j}\right)\right|_{x=0}=\left.\partial_{x} J_{s+}\left(x, \gamma ; p_{j}\right)\right|_{x=0}$ can be satisfied so that the DR (20) must be considered with $J_{f-}\left(h, \gamma ; p_{j}\right)$. Due to $a_{c}=0$,

$$
\left.\partial_{x} J_{f-}\left(x, \gamma ; p_{j}\right)\right|_{x=h}<0
$$

must hold. Thus, since only $\left.\partial_{x} J_{c+}\left(x, \gamma ; p_{j}\right)\right|_{x=h}$ is negative (see Eq.(19)), the dispersion relation

$$
\left.\partial_{x} J_{f-}\left(x, \gamma ; p_{j}\right)\right|_{x=h}=-2 \sqrt{\gamma^{2}-\epsilon_{c}} J_{f-}\left(h, \gamma ; p_{j}\right) \text {, }
$$


must be evaluated subject to PDC and condition (24) (for parameters (23)). Some solutions of the DR are represented by dispersion curves $a, b, c, d$, shown in Fig.3. Curves $a$ and $c$ are inside the regions (green) defined by the PDC and condition (24). "States" represented by curve $d$ satisfy the DR (25) but not the PDC. For $h=2$ the intensity patterns for states $A, A^{\prime}, A^{\prime \prime}, A^{\prime \prime \prime}$ are depicted in Fig.3. As outlined in the previous Section, states (for example $A^{\prime \prime \prime}$ in Fig.3) outside the green regions are not permissible as solutions of the DR. The boundaries $\alpha, \beta, \alpha^{\prime}, \beta^{\prime}$ are defined by tuples $\left\{h, \gamma, p_{j}\right\}$ that satisfy $\left.\partial_{x} J_{f-}\left(x, \gamma ; p_{j}\right)\right|_{x=h}=0$. Boundaries $\delta, \delta^{\prime}$ are given by tuples $\left\{h, \gamma_{0}, p_{j}\right\}, h_{\alpha}<h<h_{\beta}$ or $h_{\alpha^{\prime}}<h<h_{\beta^{\prime}}$, with $\gamma_{0}=$ $\sqrt{\max \left(\epsilon_{s}, \epsilon_{c}\right)}$. Hence, the cut-off values $h_{\alpha}, h_{\alpha^{\prime}}, h_{\beta}, h_{\beta^{\prime}}$ are determined as solutions of the equation

$$
\left.\partial_{x} J_{f-}\left(x, \gamma_{0} ; p_{j}\right)\right|_{x=h}=0
$$
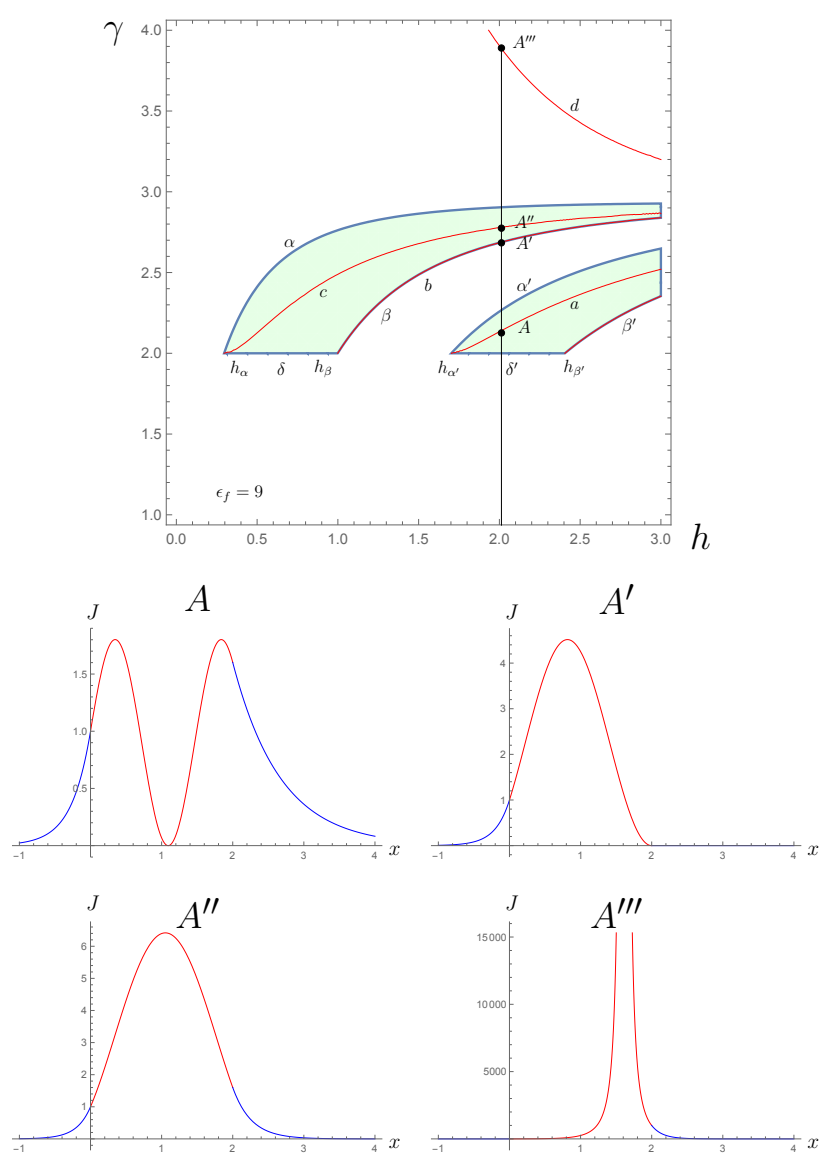

FIG. 3. $(\gamma, h)$-dispersion curves, regions (green) defined by PDC and (24), intensity profiles corresponding to physical states $\left(A, A^{\prime}, A^{\prime \prime}\right)$ and singular state $\left(A^{\prime \prime \prime}\right)$, parameters $(23)$, comments in the text.

Obviously (see Fig.3), the states $A^{\prime \prime}, A^{\prime}, A$ become (basically) unstable at $h_{\alpha}, h_{\beta}, h_{\alpha^{\prime}}$ respectively, if the thickness $h$ is decreased from $h=2$ to $h=h_{\alpha}=0.3$ (if $h<h_{\alpha}$, there is no solution at all, likewise known in linear waveguide theory).

Due to the assumed continuity of $\gamma=\gamma\left(h, p_{j}\right)$ w.r.t. $h$ and $p_{j}$ the DR (25) describes the parameter dependence of states $A, A^{\prime}, A^{\prime \prime}$. If, e.g., $\epsilon_{f}$ is variable, the corresponding dispersion curves are shown in Fig.4. 


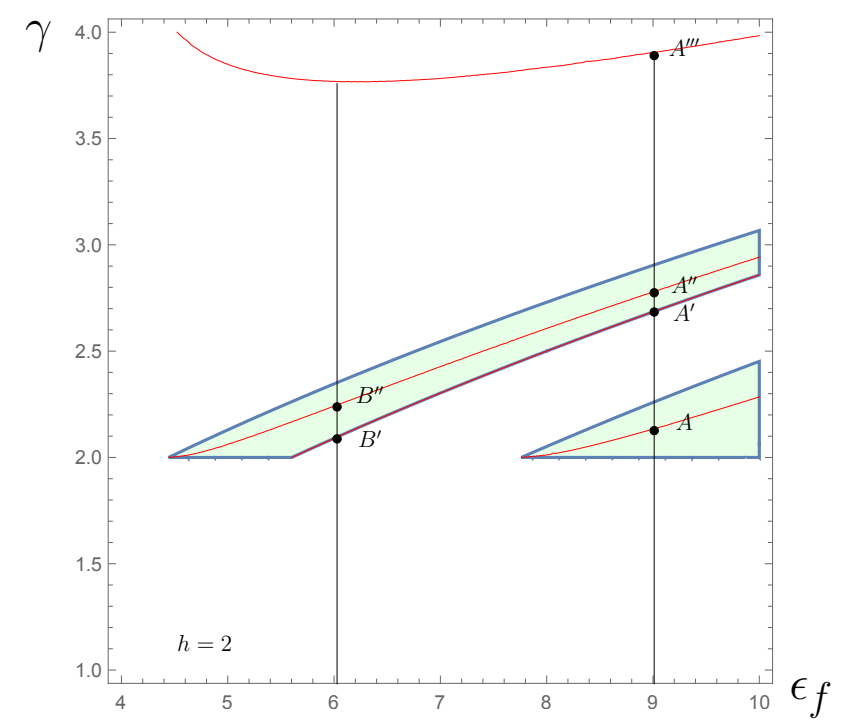

FIG. 4. $\left(\gamma, \epsilon_{f}\right)$-dispersion curves for $h=2$, regions (green) according to PDC and (24)

States $A, A^{\prime}, A^{\prime \prime}$ are stable in the vicinity of $\epsilon_{f}=9$. Selecting dielectrics with $\epsilon_{f}<7.7$, state $A$ becomes unstable. If $\epsilon_{f}<4.5$ all states are unstable. In the vicinity of $\epsilon_{f}=6$ there are two stable states $B^{\prime}, B^{\prime \prime}$ (see Fig.4). The dependence of $B^{\prime}, B^{\prime \prime}$ on $h$ is shown in Fig.5. Considering Fig.3 together with Figs.4 and 5 we can read off the stability behaviour of the states $A, A^{\prime}, A^{\prime \prime}$ due to variation of $\epsilon_{f}$ (from 9 to 6 ): $A$ becomes unstable and $A^{\prime}, A^{\prime \prime}$ remain stable. Evidently, varying $\epsilon_{f}$ in opposite direction from 6 to 9 a "new" stable state $A$ appears for $(h=2)$. States $B^{\prime \prime}, B^{\prime}$ are developing continuously to $A^{\prime \prime}, A^{\prime}$.

A similar analysis can be performed for any of the parameters $p_{j}$. If $a_{f}$ is variable, the stability of states $A, A^{\prime}, A^{\prime \prime}$ in Fig. 3 can be red off from Fig.6. In the vicinity of $a_{f}=-0.01$ the states are stable. For focussing nonlinearity $\left(a_{f}=0.01\right)$ one additional stable state $B^{\prime \prime \prime}$ is possible. The states $A, A^{\prime}, A^{\prime \prime}$ remain stable if $a_{f}$ becomes positive.

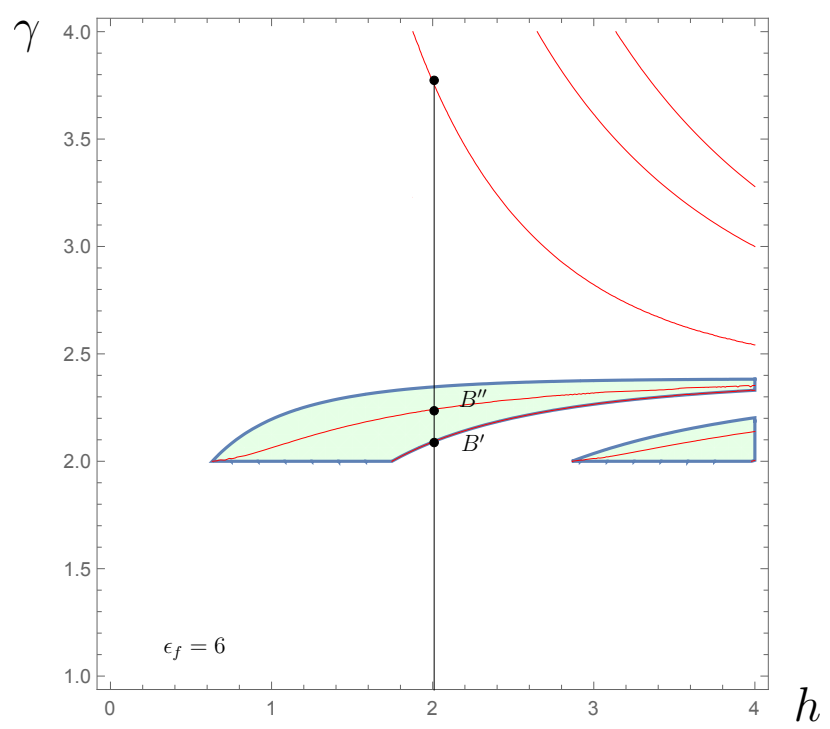

FIG. 5. $(\gamma, h)$-dispersion curves for parameters $(23)$ and with $\epsilon_{f}=6$ 
Considering state $B^{\prime \prime \prime}$ (inside the PDC region) it seems that $\gamma \rightarrow \infty$ as $a_{f} \rightarrow 0$. As outlined in Appendix B this is an artefact as can be seen from the dispersion diagram if $a_{f}=0$ (Fig.7).

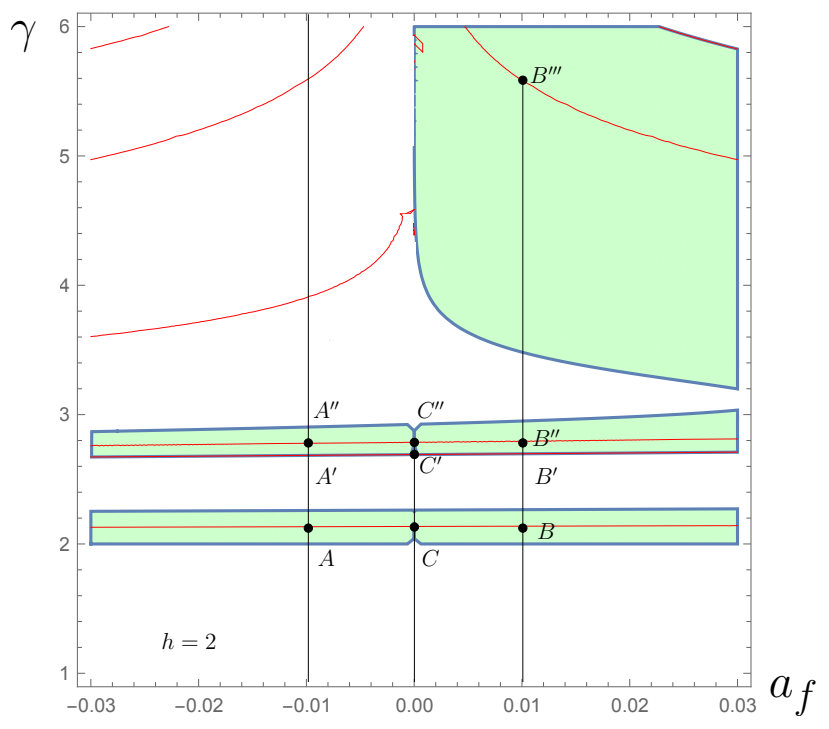

FIG. 6. $\left(\gamma, a_{f}\right)$-dispersion curves for $h=2$ and parameters $(23)$, comments in the text

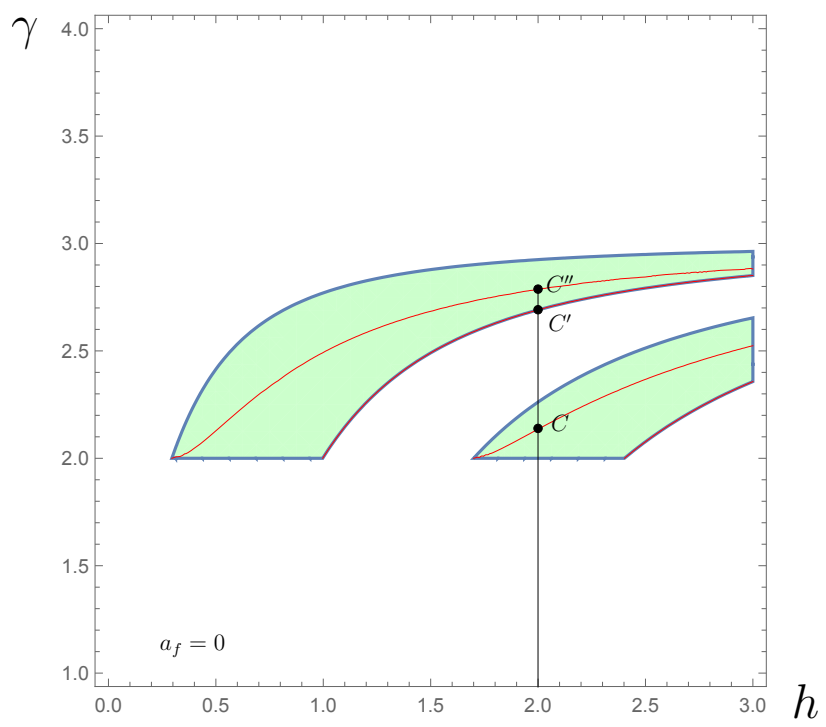

FIG. 7. $(\gamma, h)$-dispersion curves for $a_{f}=0$ and parameters (23), states $C, C^{\prime}, C^{\prime \prime}$ as in Fig.6

As a second example we consider focussing material in the film $\left(a_{f}>0\right)$. The parameters are selected as

$$
\left\{p_{j}\right\}=\left\{a_{s}=a_{c}=0, \quad a_{f}=0.02, \quad \epsilon_{f}=4.5, \quad \epsilon_{s}=1, \quad \epsilon_{c}=4, \quad J_{0}=3\right\} .
$$

The corresponding phase diagram is shown in Fig.8. DCs $a-e$ are associated to physical solutions of DR (25). Considering, for example, the dependence $\gamma\left(h_{A}\right), h_{E} \leq h_{A} \leq h_{\tilde{B}}$, of state $A$, it is obvious that instabilities occur at bifurcation state $\tilde{B}$ and at $E$. At $E$, 


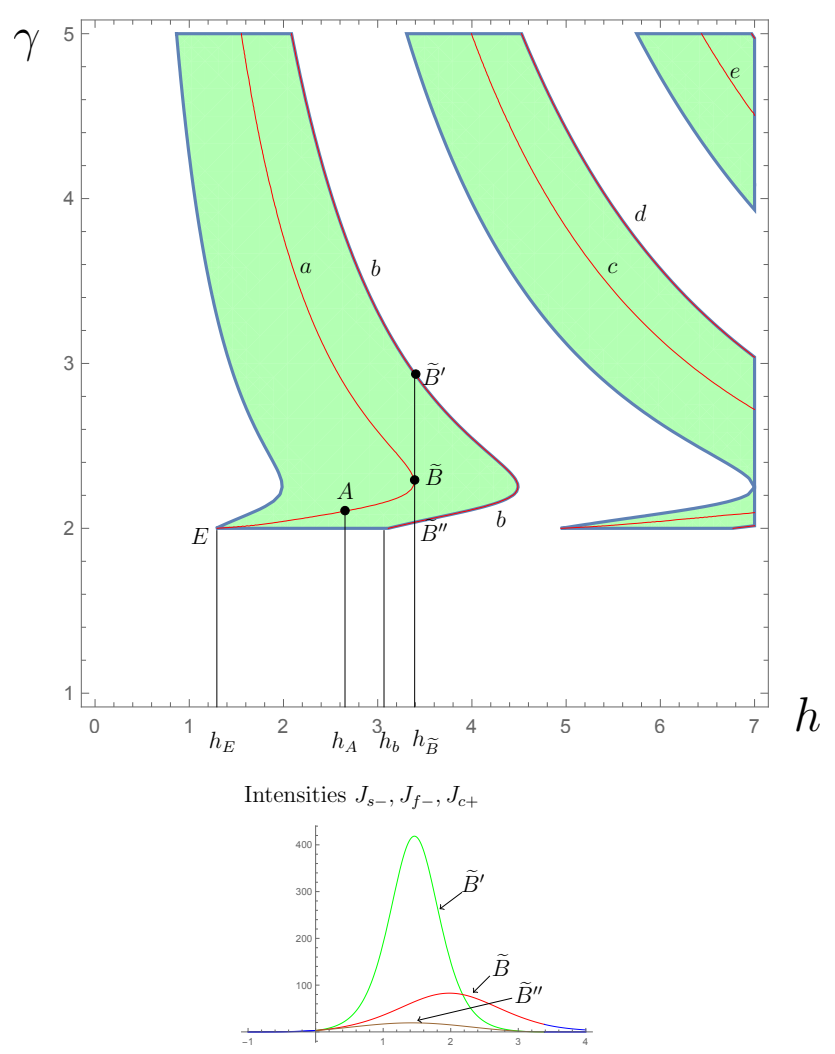

FIG. 8. $(\gamma, h)$-dispersion curves, regions (green) defined by PDC and (24), intensity profiles at $h=h_{\tilde{B}}=3.37$, parameters (27); further comments in the text

thickness $h_{E}$ is determined by Eq.(26) $\left(h_{E}=1.283\right)$. If $h<h_{E}$, there are no solutions since $\left.\partial_{x} J_{f-}\left(x, \max \left(\epsilon_{s}, \epsilon_{c}\right) ; p_{j}\right)\right|_{x=h}>0$ holds, in contradiction to $\left.\partial_{x} J_{c+}\left(x, \max \left(\epsilon_{s}, \epsilon_{c}\right) ; p_{j}\right)\right|_{x=h}<0$. If $h=h_{\tilde{B}}$, there are three (disregarding "higher" modes) solutions of the DR corresponding to states $\tilde{B}^{\prime \prime}, \tilde{B}, \tilde{B}^{\prime}$. In the vicinity of $\tilde{B}$ four states $\left(h_{b}<h<h_{\tilde{B}}\right)$ or two states $\left(h>h_{\tilde{B}}\right)$ are possible, indicating bistability w.r.t. $h$ at bifurcation state $\tilde{B}$. It is interesting to study the dependence of $\gamma$ on $\epsilon_{f}, a_{f}, J_{0}$ near bifurcation state $\tilde{B}\left(h_{\tilde{B}}=3.37\right)$. The result is shown in Figs.9-11. Remarkably, bistability w.r.t. $h$ at $\tilde{B}$ implies bistability w.r.t. $\epsilon_{f}, a_{f}, J_{0}$ that were chosen as parameters for phase diagram Fig.8. Needless to say that Figs.8-11 are consistent.

As a summary of this Section it is suitable to consider Figs.3, 7, 8 together with Fig.6 as the link between them. First we consider the transition from a nonlinear defocussing film $\left(a_{f}<0\right)$ to a linear one $\left(a_{f}=0\right)$ with $J_{0}$ selected subject to $(25)$ and the PDC. If $a_{f}<0$ (Fig.3) the PDC constrains $\gamma$ according to $\max \left(\epsilon_{s}, \epsilon_{c}\right) \leq \gamma^{2}<\epsilon_{f}-\sqrt{-a_{f}^{2} J_{0}^{2}-2 a_{f} J_{0}\left(\epsilon_{f}-\epsilon_{s}\right)}$, so that (physical) solutions $\gamma\left(h ; p_{j}\right)$ of the DR with $\gamma^{2} \geq \epsilon_{f}-\sqrt{-a_{f}^{2} J_{0}^{2}-2 a_{f} J_{0}\left(\epsilon_{f}-\epsilon_{s}\right)}$ do not exist (the roots $j_{2}, j_{2}$ are complex in this case). If $a_{f}=0$ (Fig.7) the PDC can be written as $\max \left(\epsilon_{s}, \epsilon_{c}\right) \leq \gamma^{2}<\epsilon_{f}, 0<J_{0}<\infty$, so that certain solutions $\gamma\left(h ; p_{j}\right)$ excluded if $a_{f}<0$, now exist if $a_{f}=0$. Regarding Figs. 6 and 7 states $A, A^{\prime}, A^{\prime \prime}$ are locally (w.r.t. $h, a_{f}$ ) stable $\left(A \rightarrow C, A^{\prime} \rightarrow C^{\prime}, A^{\prime \prime} \rightarrow C^{\prime \prime}\right)$ and (singular) state $A^{\prime \prime \prime}$ disappears as $a_{f} \rightarrow 0$. If $a_{f}>0$ (Fig.8) there is, according to the PDC, no upper bound for $\gamma\left(h ; p_{j}\right)$. Obviously (Fig.6), states $B, B^{\prime}, B^{\prime \prime}, B^{\prime \prime \prime}$ are locally stable, approaching $C, C^{\prime}, C^{\prime \prime}$, respectively as $a_{f} \rightarrow 0$. Since $\gamma^{2}<\epsilon_{f}$ if $a_{f}=0$, physical state $B^{\prime \prime \prime}$ (Fig.8) disappears as $a_{f} \rightarrow 0$. - The foregoing summary 
shows how parameter dependence (here w.r.t. $a_{f}$ ) and basic stability are related.

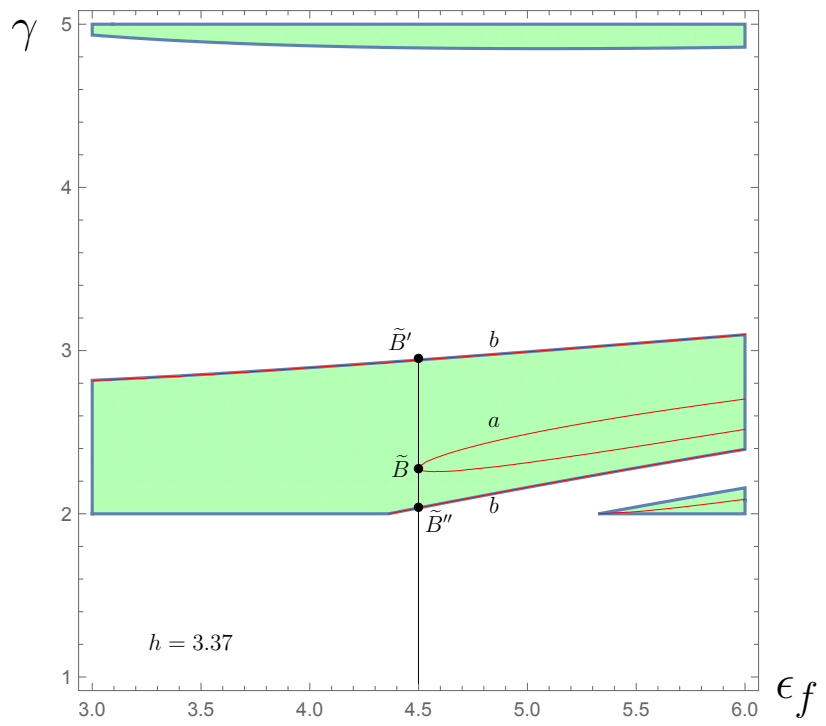

FIG. 9. $\left(\gamma, \epsilon_{f}\right)$-dispersion curves, parameters as for Fig.8

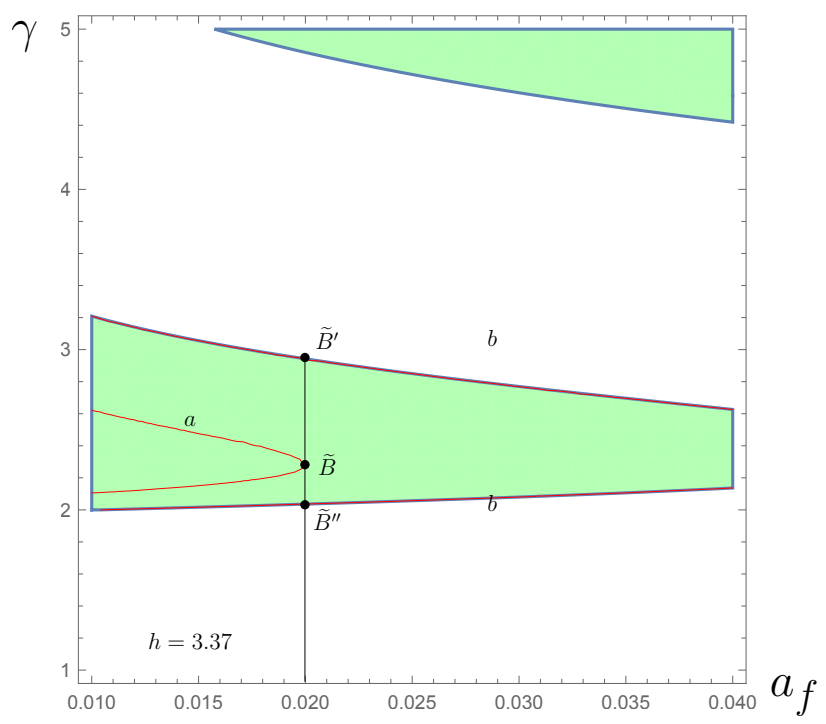

FIG. 10. $\left(\gamma, a_{f}\right)$-dispersion curves, parameters as for Fig.8

\section{CONCLUDING REMARKS}

1. Traditionally, the question of stability of nonlinear guided waves is addressed by analysing the behaviour of the perturbation (eigenfunction) $f\left(x, z, \gamma ; p_{j}\right)$ (see Section I). Since the decay properties of $f\left(x, z, \gamma ; p_{j}\right), z \rightarrow \infty$, are related to the stationary solution $E_{y}\left(x, \gamma ; p_{j}\right)$, it is useful to know the conditions for existence of $E_{y}\left(x, \gamma ; p_{j}\right)$ and the parameter dependence of $E_{y}\left(x, \gamma ; p_{j}\right)$. These conditions are derived by using the phase diagrams $\left\{\left(\partial_{x} J(x)\right)^{2}, J(x)\right\}$, associated to the cubic nonlinearity (see Fig.2). 


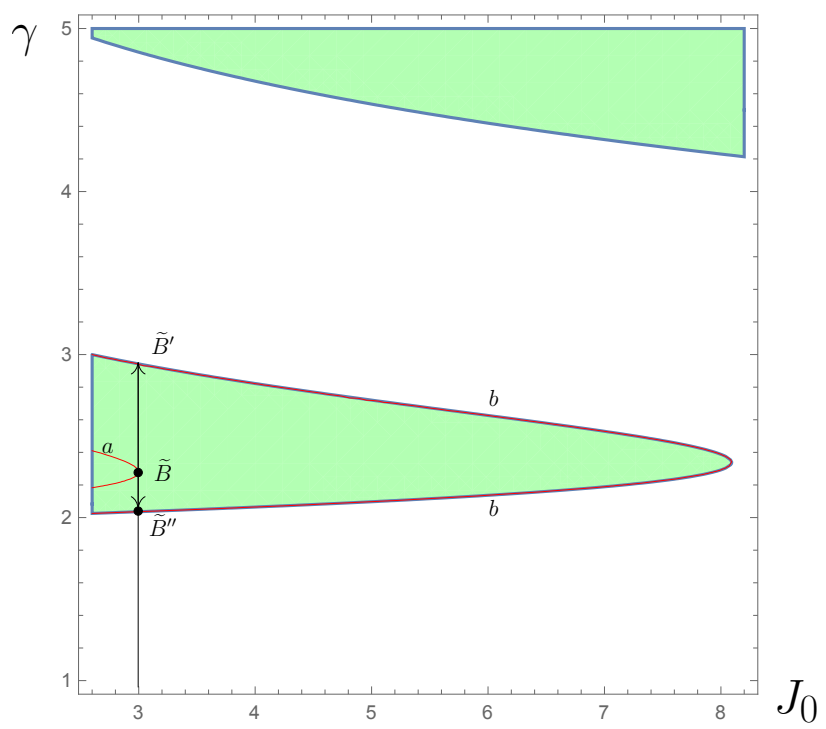

FIG. 11. $\left(\gamma, J_{0}\right)$-dispersion curves (red), parameters as for Fig.8. Arrows indicate the possibility of switching from state $\tilde{B}$ to $\tilde{B}^{\prime}$ or $\tilde{B}^{\prime \prime}$

Due to the compact representation of the intensity $J_{f \pm}\left(x, \gamma ; p_{j}\right)$ according to Eq.(9) a simple form of the DR is possible (Eq.(20)). The combination of the PDC with the DR (20) constitutes the basis for investigating the parameter dependence of solutions $\gamma=\gamma\left(p_{i} ; p_{j}\right)$ of the DR, thus associated to the parameter dependence of the stationary solution $E_{y}\left(x, \gamma ; p_{j}\right)$. Appropriately selected $\gamma$ and $E_{y}$ as "initial data" [2] can be used for a (conventional) stability analysis (with respect to propagation distance $z$ ) of $\tilde{E}_{y}(x, z, \gamma)$ as a solution of the NLSE (2). The existence of (physical) stationary solutions $E_{y}\left(x, \gamma ; p_{j}\right)$ is associated to the solutions $\gamma=\gamma\left(p_{i} ; p_{j}\right)$ of the dispersion relation (20). Based on a phase diagram analysis we have derived necessary conditions for its solutions (PDC). Due to the analytical properties of $J_{f \pm}\left(h, \gamma ; p_{j}\right)$, it is possible to check the solvability of the DR.

2. As shown in Section III, numerical evaluation of PDC and DR (20) with respect to the various parameters $p_{j}$ is straightforward (we used MATHEMATICA).

3. An explanation of the remarkable consistency of the dispersion diagrams Figs.8-11 at bifurcation points is an unsolved problem.

4. In the conventional treatment of stability usually the "initial data" are taken (see [2]) from a power $(P)$ versus $\gamma$ dispersion relation $(P, \gamma)$. The power is given by (if $a_{s}=a_{c}=0$, as assumed in Section IV)

$$
P\left(h ; \gamma, p_{j}\right)=\int_{-\infty}^{0} J_{s+}(x) d x+\int_{0}^{h} J_{f-}(x) d x+\int_{h}^{\infty} J_{c-}(x) d x .
$$

The three integrals in (27) exist (the second can only be evaluated numerically) if the PDC is satisfied. By inserting pairs $(\gamma, h)$ of a certain branch of the $(\gamma, h)$ dispersion relation into Eq. $(27)$ the $(P, \gamma)$ - dispersion relation can be obtained. This representation $P(\gamma)$ is not analytical so that it can only be studied numerically. Nevertheless it can be used for a check by means of the Vakhitov-Kolokolov stability criterion $\frac{\partial P}{\partial \gamma}>0$ despite of its restricted applicability [8]. 
5. The (simplifying) assumptions $a_{s}=a_{c}=0$ are not essential. If $a_{s} \neq 0, a_{c} \neq 0$, the intervals $\left[0, \frac{2\left(\gamma^{2}-\epsilon_{\nu}\right)}{a_{\nu}}\right]$ are finite (see Fig.2(k)) and the combination of signs in the DR (20) must be found as being exemplified for parameters (23) leading to the particular DR (25).

6. The present approach can be applied to the linear magnetic case and, in particular, to the case with both negative permittivity $\epsilon_{f}$ and permeability $\mu_{f}$ (metamaterial).

7. As far as we know the literature, the parameter dependence, and thus basic stability of the $z$-independent solution $E_{y}\left(x, \gamma ; p_{j}\right)$ has not been addressed. In this sense, the foregoing approach represents (to a certain extent) a complement to the traditional treatment of stability.

\section{APPENDIX A: THE PHASE DIAGRAM CONDITION (PDC)}

Inserting the (appropriate) PDC roots $j_{1}, j_{2} \in\left\{0, J_{1}, J_{2}\right\}$ according to (21), and $J_{1,2}^{(h)}$ according to (17), into conditions (22), these conditions can be written as

$$
a_{f}<0, \quad \epsilon_{c} \leq \epsilon_{s}<\gamma^{2} \leq \epsilon_{f}-\sqrt{-J_{0}^{2} a_{f}^{2}-2 J_{0} a_{f}\left(\epsilon_{f}-\epsilon_{s}\right)}, \quad 0<J_{0} \leq \frac{\epsilon_{s}-\epsilon_{f}}{a_{f}}
$$

or

$\epsilon_{s}<\epsilon_{c}<\gamma^{2} \leq \epsilon_{f}-\sqrt{-J_{0}^{2} a_{f}^{2}-2 J_{0} a_{f}\left(\epsilon_{f}-\epsilon_{s}\right)}, \quad 0<J_{0} \leq \frac{\epsilon_{s}-\epsilon_{f}+\sqrt{\left(\epsilon_{c}-\epsilon_{s}\right)\left(2 \epsilon_{f}-\epsilon_{s}-\epsilon_{c}\right)}}{a_{f}}$.

or

$$
a_{f}>0, \quad \max \left\{\epsilon_{s}, \epsilon_{c}\right\} \leq \epsilon_{f}, \quad \max \left\{\epsilon_{s}, \epsilon_{c}\right\}<\gamma^{2} \cup \epsilon_{s} \leq \epsilon_{f}<\epsilon_{c}<\gamma^{2}, \quad J_{0}>\frac{2\left(\epsilon_{s}-\epsilon_{f}\right)}{a_{f}}
$$

or

$$
\begin{gathered}
\epsilon_{f}<\max \left\{\epsilon_{s}, \epsilon_{c}\right\}<\gamma^{2}, \quad \epsilon_{f}<\epsilon_{s}, \quad J_{0}>0 . \\
a_{f}>0, \quad \epsilon_{f}<\epsilon_{c}<\epsilon_{s}<\gamma^{2}, \quad 0<J_{0} \leq \frac{\epsilon_{s}-\epsilon_{f}-\sqrt{\left(\epsilon_{s}-\epsilon_{c}\right)\left(\epsilon_{s}+\epsilon_{c}-2 \epsilon_{f}\right)}}{a_{f}} \\
\cup \frac{\epsilon_{s}-\epsilon_{f}+\sqrt{\left(\epsilon_{s}-\epsilon_{c}\right)\left(\epsilon_{s}+\epsilon_{c}-2 \epsilon_{f}\right)}}{a_{f}} \leq J_{0} \leq \frac{2\left(\epsilon_{s}-\epsilon_{f}\right)}{a_{f}}
\end{gathered}
$$

or

$$
\begin{gathered}
\epsilon_{f}<\epsilon_{s} \leq \epsilon_{c}<\gamma^{2}, \quad 0<J_{0} \leq \frac{2\left(\epsilon_{s}-\epsilon_{f}\right)}{a_{f}} . \\
a_{f}<0, \quad \epsilon_{s}<\epsilon_{f}, \quad 0<J_{0} \leq \frac{\gamma^{2}-\epsilon_{f}}{a_{f}} \\
\epsilon_{c} \leq \epsilon_{f}-\sqrt{-J_{0}^{2} a_{f}^{2}-2 J_{0} a_{f}\left(\epsilon_{f}-\epsilon_{s}\right)}, \quad \gamma^{2}=\epsilon_{f}-\sqrt{-J_{0}^{2} a_{f}^{2}-2 J_{0} a_{f}\left(\epsilon_{f}-\epsilon_{s}\right)} . \\
a_{f}>0, \quad J_{0}=\frac{2\left(\epsilon_{s}-\epsilon_{f}\right)}{a_{f}}, \quad \gamma^{2} \geq \epsilon_{s} \geq \epsilon_{f} .
\end{gathered}
$$




$$
\begin{array}{r}
a_{s}>0, \quad J_{0}>0, \quad \gamma^{2} \geq \frac{a_{s} J_{0}}{2}+\epsilon_{s} \quad \text { or } \quad a_{s}=0, \quad J_{0}>0, \quad \gamma^{2}>\epsilon_{s}, \\
a_{c}>0, \quad J_{1}^{(h)} \geq 0, \quad \gamma^{2} \geq \frac{a_{c} J_{1}^{(h)}}{2}+\epsilon_{c} \quad \text { or } \quad a_{c}=0, \quad J_{1}^{(h)} \geq 0, \quad \gamma^{2}>\epsilon_{c}
\end{array}
$$

or

$$
a_{c}>0, \quad J_{2}^{(h)} \geq 0, \quad \gamma^{2} \geq \frac{a_{c} J_{2}^{(h)}}{2}+\epsilon_{c} \quad \text { or } \quad a_{c}=0, \quad J_{2}^{(h)} \geq 0, \quad \gamma^{2}>\epsilon_{c}
$$

subject to Eqs.(17)-(18) with $C_{f}$ from Eq.(15).

\section{APPENDIX B: ON CONTINUITY OF $\gamma=\gamma\left(p_{i}\right)$}

Apart from an essential singularity at infinity the Figures in Section IV indicate local continuity of the relation $\gamma=\gamma\left(p_{i} ; p_{j}\right)$. We are unable to prove this mathematically. In the following we present some arguments that may serve, for practical purposes only, as an compensation for a mathematical proof. For simplicity we assume, as above, $a_{s}=a_{c}=0$ and investigate the dispersion curves $\gamma=\gamma\left(h ; p_{j}\right)$ to subject the PDC (see Fig.3). Let (see Eq. $(25))$

$$
F:=\left.\partial_{x} J_{f-}\left(x, \gamma ; p_{i}\right)\right|_{x=h}+2 \sqrt{\gamma^{2}-\epsilon_{c}} J^{(h)}\left(p_{j}\right) .
$$

A solution tuple $\bar{t}:=\left\{\bar{h}, \bar{\gamma} ; p_{j}\right\}, p_{j} \in\left\{\bar{a}_{f}, \bar{\epsilon}_{\nu}, \bar{J}_{0}\right\}$ of the DR (25) satisfies $F(\bar{t})=0$. Due to the PDC, $J_{f-}\left(h, \gamma ; p_{j}\right)$ is continuously differentiable in the neighbourhood of $\bar{t}$. Hence, $\partial_{\gamma} F(t)$ exists in the neighbourhood of $\bar{t}$. If $\partial_{\gamma} F \neq 0$ in the vicinity of $\bar{t}$ then, according to the implicit function theorem, $\gamma=\gamma(h)$ is differentiable and thus continuous. If $\partial_{\gamma} F=0$ in this vicinity, numerical evaluation shows, first, that the system $\left\{F(\bar{t})=0, \partial_{\gamma} F(\bar{t})=0\right\}$ can be fulfilled for certain tuples $\bar{t}=\left\{\bar{h}, \bar{\gamma} ; p_{i}\right\}$, and, second, that the intensities $J_{s-}(t), J_{f-}(t), J_{c+}(t)$, subject to $F(t)=0$ with $t$ in the closed vicinity of $\bar{t}$, are barely distinguishable from $J_{s-}(\bar{t}), J_{f-}(\bar{t}), J_{c+}(\bar{t})$. Thus, in general the assumption of discontinuous $\gamma=\gamma\left(h ; p_{j}\right)$ at $\bar{t}$ is not consistent with particular numerical results.

However, with regards to Fig.6, obviously there is a singularity of $\gamma=\gamma\left(a_{f} ; p_{j}\right)$ at $a_{f}=0$. But this singularity is an artefact due to the use of Eq.(9) if $a_{f}=0$. In this case $J_{f-}(x, \gamma)$ according to Eq.(9) must be replaced by

$$
J_{f l i n}(x, \gamma)=\left(\cos \left(x \sqrt{\epsilon_{f}-\gamma^{2}}\right)+\sqrt{\frac{\gamma^{2}-\epsilon_{s}}{\epsilon_{f}-\gamma^{2}}} \sin \left(x \sqrt{\epsilon_{f}-\gamma^{2}}\right)\right)^{2}
$$

since the discriminant $\Delta$ of Weierstrass' elliptic function $\wp\left(x ; g_{2}, g_{3}\right)$ is equal to zero [24]. In DR $(25), J_{f l i n}(x, \gamma)$ must be used in place of $J_{f-}\left(h, \gamma ; p_{j}\right)$ leading to Fig. 7 that does not show a singularity. If $\Delta=0$ for other parameters $p_{i}$, similar pseudo-singularities as for $a_{f}=0$ may occur.

To sum up, it seems justified to consider $\gamma=\gamma\left(p_{i} ; p_{j}\right)$ as "practically" continuous though a rigorous mathematical proof is missing.

The authors would like to thank Dr. Markus Harju, University of Oulu (Finland), for useful discussions and for preparing the figures. 


\section{REFERENCES}

* hwschuer@uos.de

† vserov@cc.oulu.fi

[1] P: N. Butcher and D. Cotter, The elements of nonlinear optics, (Cambridge Univ. Press, Cambridge, 1991), p. 216.

[2] C.K.R.T. Jones and J.V. Moloney, Physics Letters A, Vol. 117, pp. 175-180 (1986).

[3] A.D. Boardman, P. Egan, T. Twardowski and M. Wilkins, in Nonlinear Waves in Solid State Physics, edited by A.D. Boardman et al. (Plenum Press, New York, 1990).

[4] H.T. Tran, J.D. Mitchel, N.N. Akhmediev and A. Ankiewicz, Optics Communications, Vol. 92, pp. 227-233 (1992).

[5] N.N. Akhmediev, in Nonlinear Surface Electromagnetic Phenomena, edited by H.-E. Ponath and E.I. Stegeman (Noth-Holland, Amsterdam, 1991), p. 316.

[6] J.V. Moloney et al., Applied Physics Letters, Vol. 48, pp. 826-828 (1986).

[7] N.N. Akhmediev, A. Ankiewicz and H.T. Tran, J. Opt. Soc. B, Vol. 10, pp. 230-236 (1993).

[8] H.T. Tran, Optics Letters, Vol. 17, pp. 1767-1769 (1992).

[9] L. Tran et al, Optics Letters, Vol. 20, pp. 2183-2185 (1995).

[10] J. Miranda, Phys. Rev. A, Vol. 46, pp. 5999-6001 (1992).

[11] A.S. Reyna, Phys. Rev. A, Vol. 92, pp. 33810-33815 (2015).

[12] D. Mihalache and M. Mazilu, Solid State Communications, Vol. 63, pp. 215-217 (1987).

[13] K. Oguso, Optics Communications, Vol. 83, pp. 260-264 (1991).

[14] H.T. Tran and A. Ankiewicz, IEEE Journal of Quantum Electronics, Vol. 28, pp. 488-492 (1992).

[15] A.V. Buryak and N.N. Akhmediev, Phys. Rev. E, Vol. 51, pp. 3572-3578 (1995).

[16] D.J. Mitchell and A.W. Snyder, J. Opt. Soc. B, Vol. 10, pp. 1572-1580 (1993).

[17] Y. Chen and J. Atai, IEEE Journal of Quantum Electronics, Vol. 31, pp. 2095-2100 (1995).

[18] W. Chen and A.A. Maradudin, J. Opt. Soc. Am. B 5, p. 529 (1988).

[19] (a) H.W. Schürmann, Z. Phys. B 97, p. 515 (1995); (b) H.W. Schürmann, V.S. Serov, and Yu.V. Shestopalov, Phys. Rev. E, Vol. 58, pp. 1040-1050 (1998).

[20] L. Gagnon and P. Winternitz, J. Phys. A, Vol. 22, p. 469 (1989); H.W. Schürmann, Phys. Rev. E, Vol. 54, pp. 4312-4320 (1996).

[21] Here, $p_{i}$ denotes variable parameter and $p_{j}$ the fixed parameters.

[22] (a) P.G. Drazin and R.S. Johnson, Solitons: an Introduction (Cambridge University Press, Cambridge, 1989), pp. 22-25; (b) H.W. Schürmann and V.S. Serov, Phys. Rev. A, Vol. 93, 063802 (2016).

[23] E.T. Whittaker and G.N. Watson, A Course of Modern Analysis (Cambridge University Press, Cambridge, 1927), p. 454.

[24] M. Abramowitz and I.A. Stegun, Handbook of Mathematical Functions (Dover, New York, 1965), p. $652(18.12 .27)$. 